\title{
Effect of Routing Protocols and Layer 2 Mediums on Bandwidth Utilization and Latency
}

\author{
Ghulam Mujtaba ${ }^{1}$, Furhan Ashraf ${ }^{3}$, Fiaz Waheed ${ }^{4}$ \\ Department of Computer Science \\ GC University Faisalabad \\ Faisalabad, Pakistan
}

\author{
Babar Saeed ${ }^{2}$ \\ Department of Switching \\ National Telecom Corporation \\ Faisalabad, Pakistan
}

\begin{abstract}
Computer networks (CNS) are progressing as emerging field in information and communication technology (ICT). Various computer networks related problems relies on performance of computer network specifically bandwidth utilization and network latency issues. CNS especially Routing protocols play a vital role for management of network resources as well as for managing the network performance but on the other hand these have adverse effect on performance of network. Network routing protocols, bandwidth and latency rate of any computer network are tightly bounded with each other with respect to network performance. This research is being conducted to analyze the relationship between performance of different protocols, their effect on bandwidth utilization, and network latency rate using layer 2 medium. After analysis of relationship of these parameters suggestions will be made for enhancement of network performance over layer 2 medium.
\end{abstract}

Keywords-Routing protocols; layer2 technologies; FDDI; latency rate; bandwidth utilization

\section{INTRODUCTION}

In this emerging era of ICT technology, usage of multimedia devices are growing immensely, so computer network infrastructures and architectures are also being developed in rationalized manners. This rapid change in network technology caused for diversity in network architecture and traffic that introduced various issues those address to network performance. These issues are related to transportation of data from source to destination, data security, data size, speed and Packet loss. Other observed issues in newly developed networks are increased bandwidth utilization and packet latency rate that invoked due to the diverse network architecture and traffic. These prompted issues are required to resolve for better communication over the internet especially when multimedia contents are required to transport over computer networks.

Network routing protocols are base for transportation of data packets from source node to destination node using various alternative available routing paths. Routing protocols like RIP (Routing Information Protocol), OSPF (Open Shortest Path First), BGP (Border Gateway Protocol), EGP (Exterior Gateway Protocol) and IS-IS (Intermediate System to System Protocol) are used for efficient, dynamic and intelligent data packet transportation over the network. The major issues while deploying these routing protocols are extra bandwidth utilization and increased packet latency rate occurred due to the routing overhead traffic of these routing protocols. This wobbly behavior of routing protocols and routing overheads are cause for low throughput, higher network delay and degradation of multimedia application performance [1]. These problems can resolved through efficient bandwidth utilization, reduction in network delay, packet latency rate and through controlling routing protocols overhead traffic.

Router in network architecture acts as intermediate device of all connected networks and plays a vital role for communication and transportation of data. Routers over network layer can access every type of information transported in packet stream like L2, L3 routing information, Application Information and packet header information [2]. Start of the art revolution in network and communication technology and demand of new network services enforce development of routing protocols, transport technologies and management of network resources like bandwidth and latency time for increasing network performance [3].

Recently invented network routing techniques focused on system requirement, network extensions, topology change, data delivery, quality of service and cost effective management of network resources. Researchers adopts packet lost and packet latency rate techniques for estimation of network performance in which total packet lost percentage and RTT (Round Trip Time) of a packet is observed for evaluation of network performance. Physical topology of network is also key factor that can affect network performance. Issues of network topology can cause performance degradation of network hence in the presence of most advance routing protocols [4]. As discussed previously routing protocols are vital role player for transportation of packet delivery from source node to destination node but all routing protocols do not have capability to resolve the packet latency and packet loss issues due to their own overhead traffic, network convergence time and exchange of routing information. Numerous routing protocols are available to deploy but all routing protocols are not well admired for resolving the latency and bandwidth issues because every routing protocols have diverse way of communication over the network, so selection of best routing protocol is main consideration for better network performance [5].

Keeping in view the TCP/IP model that is basic communication model deployed in network communication, packet from Application layer to Physical layer traverse and each layer include its information along with user's data-gram. Network layer or IP layer is responsible for transportation of data packets from end to end nodes using IP address. Routers are devices over IP layer those are responsible for connecting end devices with IP addressing and also responsible for availability of alternative paths from source to destination. This network layer have a list of routing protocols compatibility with diverse 
attributes of every routing protocol, their architecture, way of communication, methods for networks convergence and selection of best fit path from source node to destination node. Data packets travel on network layer included network layer header which contain information or source address and destination address. Data packets traversed from network layer handed over to data link layer which is responsible for framing of data packets received from network layer. Technologies at layer 2 or Medium Access Control layer are Ethernet, ATM, FDDI, Cell Relay and Frame Relay. Ethernet, FDDI and other Layer medium technologies are also known as Local Area Network technologies. Ethernet is most flexible, cost effective and fast packet transmission technology. Ethernet medium access control layer technology can deployed through wired or wireless infrastructure respectively in LAN and WLAN. Ethernet designed by DEC (Digital Equipment Cooperation) adopts MAC address based transmission of data frames and broadcasts data frames over the network [6].

Asynchronous Transfer Mode (ATM) is another Medium Access Control layer transport technology that is used for transportation of data frames over the layer 2 network. ATM transport data frames in the form of fixed length cells where every cell contain pay load and header data with length of 53 octets. Fixed length cell of 53 octets contains 48 octets of user information and 8 octets of ATM control information. As opposed to Ethernet, ATM transport technology transmit data frames over fixed dedicated end to end connection oriented paths those are defined though VPIs (Virtual Path Indicators) and VCIs (Virtual Circuit Identifiers) [7]. Due to dedicated path and connection oriented communication ATM is deployed to get a satisfactory performance of computer networks. A protocol stack with ATM adaptation layer name is designed for communication using ATM.

FDDI (Fiber Distributed Data Interface) is also layer 2 medium access control transportation mechanism that transmits data packets with diverse data rate from $100 \mathrm{Mbps}$ to $1 \mathrm{Gbps}$ over the Fiber Optic medium. FDDI used for connecting Ethernet or Token ring networks with each other using FDDI provisioned Switches and hubs and also deployed for high speed application networks with diverse network topologies like Ring, Star and Tree network topology. FDDI deployed for construction of large scale robust computer networks with numerous advantages over other layer 2 technologies like area of coverage, number of nodes and cost effective. FDDI can deploy using Multi-mode, Single Mode and even using the copper wire UTP and STP cables used for connectivity of computer nodes over LAN with provision of $100 \mathrm{Mbps}$ to $1000 \mathrm{Mbps}$ of data rate.

\section{Classification of Routing Protocols}

Routing protocols can classify based on their structure, design, operational principal and inter-communication mechanism. These classifications can bifurcate as fixed design, flexible design and base design or default design of routing protocols. Three major classes of routing protocols based on their operational structure are as following: A- Distance Vector Routing Protocols B- LINK STATE Routing Protocols CComposite and Hybrid Routing Protocols

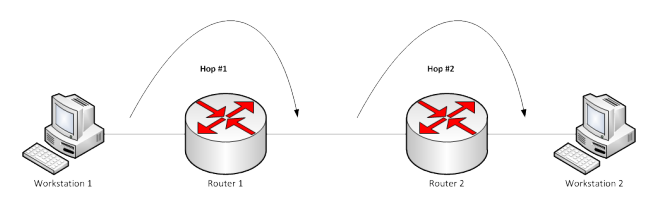

Fig. 1. Distance vector [7]

\section{A. Distance Vector Routing Protocols (DVRPs)}

Distance Vector Routing Protocols (DVRPs) Routing protocols designed with Distance vector algorithms are knows as Distance Vector Routing Protocols. Distance Vector routing protocols required information of intermediate routing hardware devices to calculate the distance from source node to destination node (Fig. 1). On the basis of intermediate routing devices such routing protocols determines the best fit path between source node and destination node. Route prioritization mechanism on the basis of distance is performed and available routing paths are stored in routing table which is adopted on failure of selected route for transportation of data packets. For calculation of best available route from possible available routing paths Bellmen-Ford and Ford-Fulkerson algorithms are incorporated to construct these distance vector routing protocols. RIP version 1 and RIP Version 2 are considered as distance vector routing protocol of local domain [8]. These distance vector routing protocols are deployed as interior routing protocols and not considered well operational for large scale of networks. RIP shares routing information in the form of routing table through broadcasting its routing information with adjacent routers after $30 \mathrm{sec}$ of time interval. The major restriction for RIP is its limited number of hops that is 15 due to which it is avoid deploying for large networks.

\section{B. LINK-STATE Routing Protocols}

As discussed earlier operational fundamentals of distance vector routing protocols are distance and vector. Intermediate routing devices also known as hops are considered the base for calculation of distance from source node to destination node. On the other hand link state routing protocols as showed in Fig. 2 instead of calculating the distance construct a topological map based on network topology and connected routers in the network. Link state advertisements are sent by each node to its adjacent nodes on which basis every node construct its topology map. Once topology map on each node has been constructed through advertised link state messages every node in the network runs Dijkstra algorithm for calculation of shortest path from source node to destination node on the basis of link cost of each path through its available bandwidth size and other parameters.

Hello packets are examples of routing information exchange by LINKSTATE routing protocols but in LINKSTATE routing protocols these routing information to adjacent routers are sent only when any topological change occurred. Link State routing protocols prevent to change each routing table and information of each connected routed and its links are provided to directly connected routers. Every router running with OSPF discover its adjacent router and exchange information about discovered router with other adjacent routers using link state 


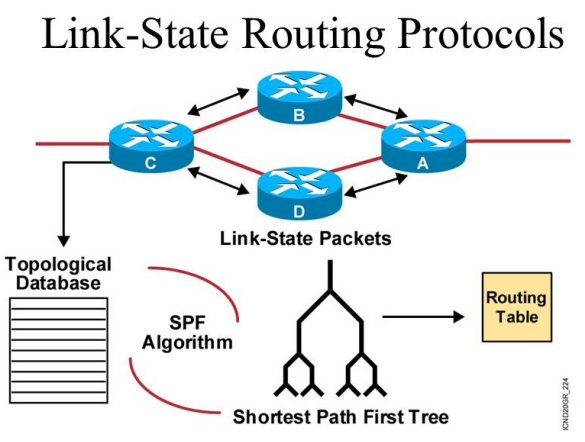

Fig. 2. LINKSTATE routing technique [9]

packets [4]. After interval of 30 seconds selective routing information is exchanged with adjacent routers, on the basis of which destined routers update their routing tables such routing information containing packets are known as LSP packets [10].

\section{Hybrid/Composite Routing Protocols}

Hybrid or Composite Routing Protocols are class of routing protocols those share the characteristics of Distance Vector and LINKSTATE Routing Protocols. Such protocols select best fit routing path among all available possible routing paths after estimation of distance and status of the links. Hybrid Routing protocols are considered as low power and memory consuming routing protocols with optimum routing performance [12]. Distance Vector Routing Protocols calculates routing paths on the basis of distance and directions vector functions and distance is considered as hop count while direction is determined through interfaces.

EIGRP is considered as Hybrid or Composite Routing protocol with characteristics of both Distance Vector and Link State Routing protocols. EIGRP is CISCO proprietary protocol that means it only runs on CISCO routers and also known as upgraded version of IGRP (Interior Gateway Routing Protocol). For routing path selection priority EIGRP is equipped with Diffusion Update Algorithm (DUAL). Easy configuration, loop prevented routes, backup paths to destined networks, low convergence and bandwidth utilization and support for VLSM (Variable Length Subnet Mask) and Classless Inter Domain Routing (CIDR) are advantages offered by the EIGRP. For route calculation bandwidth, delay, reliability, load and MTU (Maximum Transmission Unit) parameters are considered by EIGRP [13].

\section{PERFORMANCE MEASURING PARAMETERS}

Performance measuring are parameters selected for evaluation of performance of selected routing protocols with collaboration of layer 2 medium. On the basis of these matrices performance of routing protocols with collaboration of layer 2 medium can be evaluated and conclusion can be made that which routing protocols performances best with which layer 2 medium technologies. For evaluation of network convergence, network convergence activity and network convergence time parameters are selected. For evaluation of bandwidth utilization of routing protocols and routing over heads routing traffic sent and received by routing protocols will be observed.
With respect to packet latency parameters like end to end delay and packet loss will be monitored. On the basis of above mentioned performance measuring parameters network convergence, bandwidth utilization and packet latency of three selected routing protocols will be observed and decision for best routing protocol with collaboration of layer 2 medium will be decided. Detailed description of selected performance measuring parameters is given below

\section{A. Bandwidth Utilization (Bits/Sec)}

Routing protocols selected for performance comparison with collaboration of layer 2 mediums like ATM, FDDI and Ethernet sends their routing information to their adjacent routers for network convergence and propagation. Once network convergence performed application data is sent from source node to destination node. Routing protocols RIP, OSPF and EIGRP periodically broadcast their routing table for exchange of information regarding routing to their adjacent routers. This routing information is considered as routing over head and use bandwidth due to which used data is affected. Routing Traffic Sent/Received are parameters those are used for monitoring which routing protocol utilize more bandwidth for sharing of routing information and utilize extra bandwidth.

1) Routing Traffic Received (Bits/Second): Routing Traffic Received is performance evaluation parameters that describes about the routing traffic of routing protocol received from its adjacent routers that carries routing table information of any neighbor router. This parameter measures received routing traffic in bit/second.

2) Routing Traffic Sent (Bits/Second): Routing Traffic Sent is also performance measuring parameters for calculation of routing information sent by the routers to its adjacent routers. This parameter also calculates routing traffic sent by the router in Bit/Second. Through generated graphs of routing information sent it can be analyzed which node in the network sends how much routing information to other connected routers and how much bandwidth utilization from the routing protocols is consumed.

\section{B. Network Convergence Process}

Network convergence process is performed over network when all routers in network are configured with identical routing protocol because every routing protocol has its own communication architecture. Routers in network exchange routing information with adjacent routers for construction of network topology and for this purpose every router in network broadcast its routing table information to neighbor routers. After propagation of routing table information every router in network have information about source to destination and possible available paths. The reason for not using convergence by the exterior gateway routing protocols is the presence of the internet that is the well enough fast for the communication. Two main parameters network convergence activity and network convergence time are used for evaluation of network convergence process of selected routing protocols.

1) Network Convergence Activity: At the start of network convergence activity every router in network exchange its routing table information with other routers to keep them acknowledge about the available path through that router. 
In case of network topology change occurred information regarding topological change is also exchanges with adjacent router in network and routing information on basis of received information is propagated. Network Convergence activity can help to estimate the convergence capability of routing protocols and elaborate how much quickly routing protocols can converged network topology and manage change in network.

2) Network Convergence Duration (Seconds): Network convergence as discussed above is activity of routing information sharing among routers connected in network and the network convergence time is time that any routing protocol takes to converge the network and propagate routing information to all over the router in network. The efficiency of working depends upon the fast reaching of the routers on convergence but network volume is considered as constraints for network convergence time. Different protocols have different capability with respect to network convergence [14]. RIP is considered slower protocol with respect to network convergence time while OSPF is faster than the RIP. OSPF has the ability to converge in seconds.

3) End to End delay (M/Seconds): When a packet is transmitted by source node in network until receiving transmitted packet to destination node, the time packet spend on network is considered end to end delay. Networks with larger end to end delay are considered more packet loss prone networks. Various causes can cause for large end to end delay of network like congested links, buffer overflow, slow processing speed of network nodes and higher bandwidth utilization of links. Higher end to end delay can affect the performance of network and in worse condition a larger packet drop also can be observed.

4) FTP Download/Upload Response Time: FTP (File Transfer Protocol) is selected as application for transportation of application packets. Client Server architecture of FTP is designed in which a server is equipped with FTP service and 50 client nodes request to FTP server for FTP file download and upload. FTP download and upload response time is performance measuring parameters that observes how much time a server or client takes to respond for upload or download of a file. FTP Download and upload response time can affect due to the higher utilization of links, end to end delay. This parameters is selected for observing the behavior of selected routing protocols and layer 2 medium when using with collaboration.

5) Ethernet Delay: A packet transmitted by a node from source to destination traverse through different layers of TCP/IP protocol and each layer performs its functionality on its own. Layer 2 medium access layer of TCP/IP protocol deals with different protocols for framing of transmitted packet when received from network layer. ATM, Frame Relay, Ethernet, FDDI are layer 2 of medium access control technologies. Ethernet Delay is observed for observing the delay that packet takes on layer 2 of TCP/IP.

\section{Research Question to BE RESOlVED}

1) Which routing protocol from three selected routing protocols work efficiently with medium access layer technology ATM, FDDI and Ethernet?
2) Which routing protocol utilized higher bandwidth as overhead and which layer 2 transmission technology supports to routing protocols for enhancement of its performance?

3) What is the effect of routing protocols and layer 2 medium technologies over packet latency rate and bandwidth utilization?

4) How selection of layer 2 transmission technology with routing protocols can cause for reduction of network latency and bandwidth utilization overheads?

The remaining areas of this paper are organized as follows. Firstly brief overview of related work is presented. In Material and Methodology section simulation models and scenarios are discussed in detail. Result and Discussion sections includes graphical results of simulation according to performance measuring parameters. Summary contains in depth analyses of results and provide conclusion of research.

\section{RELATED WORK}

Filipiak et al. (2002) Explained numerous options of routing architectures for ATM (Asynchronous Transfer Mode) medium access control network layer protocol based on newly invented User/Network Interface (UNI) protocols. Characteristics of these User/Network Interface protocols include bandwidth reallocation, routing updates and call connection levels which distinguish this protocol. Corresponding procedures of functions are characterized with respect to prerequisites of traffic performance and regarding ATM (Asynchronous Transfer Mode) protocol layers architecture. Asynchronous Transfer Mode node architecture is also defined in detail [15].

Ioan et al. (2013) stated that Network layer of OSI model performs functionality of packets routing from source node to destination node in network. Route selection and data structure of routed packets depends on algorithms executed on network layer. This paper examines performance of three main routing protocols RIP, OSPF and EIGRP specifically for Video, HTTP and voice applications. Behavior of selected routing protocols is also inspected when link failure or recovery is occurred between network nodes. Through network simulation performance of routing protocols is analyzed and compared and their effectiveness and performance over the implemented network is studied [16].

Sheela et al. (2010) studied that which routing protocol is best choice to be implemented among distance vector, link state and hybrid routing protocols. On the basis of diverse performance comparison parameters and in depth simulation study it is claimed that EIGRP offers superior network convergence time, effective memory and CPU utilization with least bandwidth utilization requirement compare to OSPF and RIP. RIP, OSPF and EIGRP all three selected routing protocols from diverse families are considered dynamic routing protocols being deployed in real life computer networks for propagation of network topology information to adjacent routers. A list of static and dynamic routing protocols is available but selection of most effective routing protocol for network routing based on diverse performance parameters critically relevant to convergence, scalability, memory and CPU utilization, security and bandwidth utilization requirements [17]. 
Omitola et al. (2014) stated that performance evaluation of network technologies possible by the help of certain performance measuring parameters like throughput and delay, experiences of one user to other users. The behavior and performance of network technologies affected by node or user density and certain generated parameters. This research work is based on evaluation and investigation of effect of throughput and delay on two selected layer 2 technologies Ethernet and FDDI. Researcher used two scenarios each with node density 20 simulated with Opnet Modeler simulator. Both selected technologies adopt data transmission speed of 100Mbps and designed scenarios are evaluated by the output generated graphs. Results concluded that throughput in Ethernet network technology is greater than FDDI and Ethernet networks are more delay prone than FDDI with same node density [6].

Mark et al. (2017)described that the modern build centers of data provide the maximum outcome from all of the stack holders like the high bandwidth, low latency and the best usage of the topologies exists. But the problem comes in the way is the transport protocol and the not the best delivery of the data present. The researcher purposed the architecture named NDP novel based data center protocol architecture. That has the capability to deliver the data in different scenarios and different condition in a wide range of data in an efficient manner. There are the many of the buffers used in the purposed architecture NDP and these are used to the deliverance of the data in the priority manner. The priority numbers stored there in the headers of the buffers and then the buffers used their header to do the work. So by using this buffers there is the full view of the system present and made the performance efficient. So the timescale used there for the management of time named RTT. The software used named DPDK and the hardware used there named NETFGA system. So all of the usages of these things made the system performance more and better. So there is the evaluation of the system also there [18].

\section{Material And Methods}

For this research work Opnet Modeler 14.5 is used for design, configuration and execution of network topology. Various network simulations ranging from commercial to open source are available in market for computer networks research purpose. OPNET Modeler is high level commercial network design and configuration tool that help for studying computer networks at packet level and their analysis. OPNET Model is discrete simulator that observe each and every discrete event occurred during the execution of simulation and records all activities relevant to packet transmission from source to destination [19]. OPNET modeler provides support for heterogeneous networks simulation design configuration and variety of network supporting protocols of TCP/IP and telecom.

Other available network simulators are NS-2, Qualnet, GlomoSim, OMnet++ used for network design and configuration and equipped with diverse attributes and characteristics. Performance of routing protocols, diverse layer 2 technologies, MANETs, Adhoc Networks, Telecom Networks, Optical Fiber Networks and other technologies can be evaluated through discrete event simulations though OPNET Modeler [20]. Some most frequently used network simulators and their characteristics are elaborated below.
TABLE I. LIST OF SIMULATORS AND THEIR PROPERTIES

\begin{tabular}{|l|l|l|l|l|}
\hline Simulator & Commercial Open Source & Educational Support & Simulation Type & Protocol Supported \\
\hline OPNET & Commercial & Yes & DES, Object Oriented & ATM, MANET, FDDI, Wi-Fi, TCP \\
\hline Qualnet & Commercial & No & Distributed and Parallel & Wired, Wireless, WLAN etc. \\
\hline NS-2 & Open Source & Yes & Library-Based Parallel & Wireless Network \\
\hline NS-3 & Open Source & Yes & DES, Object Driven & Multicast routing, TCP, MANET, Wireless etc. \\
\hline OMNet & Open Source & Yes & DES Modular Component Based & Wireless Network \\
\hline
\end{tabular}

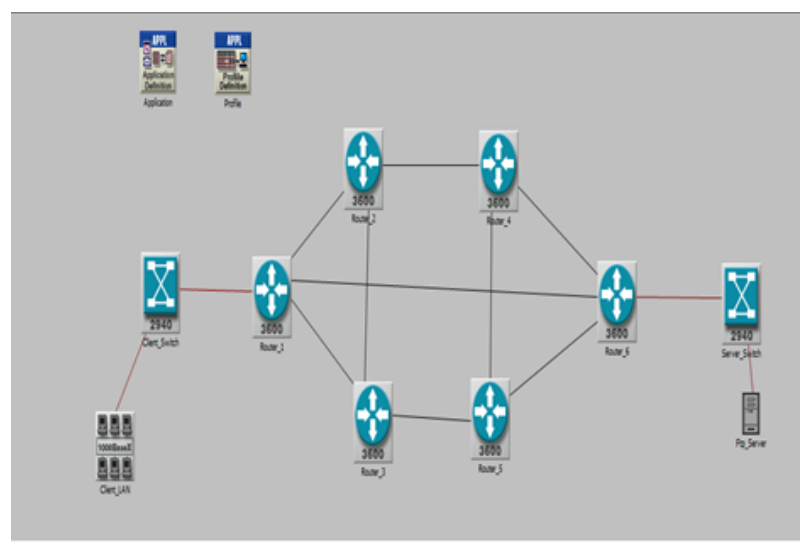

Fig. 3. Network Simulation Model

As discussed in Table 1 that three routing protocols are selected for evaluation along with three layer 2 technologies. Routing protocols are RIP, OSPF and EIGRP which are selected from three main categories of routing protocols distance vector, Link State and Hybrid. ATM, Ethernet and FDDI choose as layer 2 transportation technologies. For evaluation of routing protocols with collaboration of layer 2 technologies, scenarios are designed for each routing protocol with all three selected layer 2 transport technologies. Total numbers of 9 scenarios are design in which RIP, OSPF and EIGRP are configured with ATM, FDDI and Ethernet. After execution of simulation generated result graphs for selected performance measuring parameters are saved and analyzed.

For performance evaluation of routing protocols and layer 2 medium technologies performance comparison parameters Routing Traffic Sent/Received, Network Convergence Activity, Network Convergence Duration, End to End delay and Ethernet delay are monitored. Core networks are configured with routing protocols RIP, OSPF and EIGRP and CISCO router as devices are selected while at layer 2 medium devices for each technology FDDI, ATM and Ethernet are deployed as per standard relevant to these technologies. For Ethernet layer 2 network Ethernet switches, for ATM network ATM switches and FDDI switches for FDDI network are deployed. For packet transmission over the network FTP is used as application and a server client architecture model is deployed for FTP application. At client side network Ethernet LAN model is deployed which have $50 \mathrm{ftp}$ clients and on destination ftp server with FTP application is configured for receiving FTP requests from client nodes.

FTP Application Node is configured for high load ftp for transmission of large ftp file from server to client and client to server. OC-1 Links with data rate of $2 \mathrm{Mbps}$ for connectivity of core networks in scenario are used while for connectivity of layer 2 devices switches, Ethernet LAN and FTP Server 100Mbps Ethernet links are configured. 


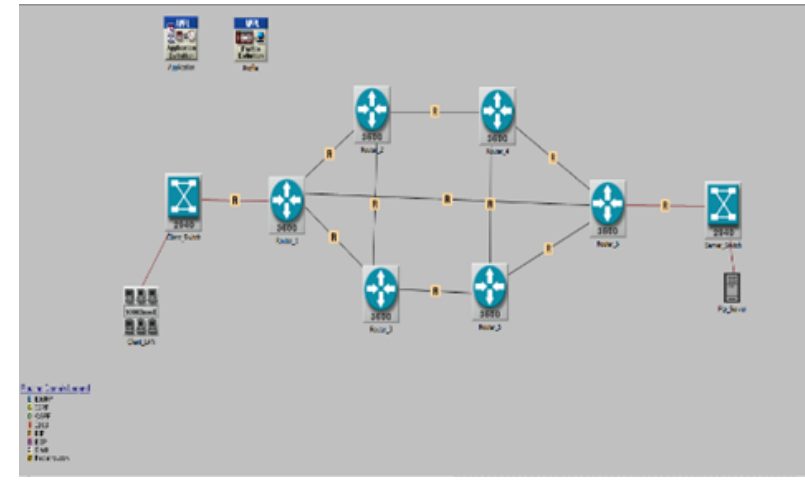

Fig. 4. RIP with Ethernet

\section{A. Routing Protocol Scenarios with Layer 2 Technologies}

Network scenarios for three selected routing protocols RIP, OSPF and EIGRP with three selected layer 2 technologies are created as shown in Fig. 3. A total number of 9 scenarios created for the evaluation of routing protocols performance with ATM, FDDI and Ethernet layer 2 technologies Each scenario consist of 6 core router that develop a core layer 2 network and at both sides Layer 2 networks are connected. Three scenarios for RIP routing protocol with FDDI, ATM and Ether are created and same like that OSPF and EIGRP scenarios with above mentioned layer 2 technologies are constructed.

1) RIP Scenario with Ethernet: Fig. 4 depicts construction of RIP routing protocol scenarios with Ethernet layer 2 technology. In this scenario core router are configure with RIP version 1 routing protocol while on layer 2 Ethernet technology is configured. Layer 2 links are 1000baseX Ethernet links those support transmission rate of $1000 \mathrm{Mbps}$ with Ethernet packets framing. Ethernet packets are variable length packets. At layer 2 Ethernet Switches with connectivity of Ethernet LAN and FTP server are configured. Ethernet LAN contain 50 Ethernet workstation each configured with FTP high load application for transmission of FTP packets. Catalyst 2948G CISCO switches are used in the simulation at both client and server ends.

2) RIP Scenario with FDDI: Fig. 5 shows RIP scenario with FDDI layer 2 technology that is token based network technology. FDDI links are configured at layer 2 with FDDI devices these FDDI links sends traffic in optical form with transmission speed of 1000Mbps. Switch devices at layer 2 also have support for connectivity of FDDI links. Rest of the objects are used same as used in above simulation.

3) RIP with ATM: Fig. 6 shows design of RIP routing protocol with ATM layer 2 technology. Only layer 2 devices and links are replace with respect to ATM technology other parameters and objects are same as designed for above mentioned RIP scenarios.

Above mentioned three scenarios are configured for RIP, same as above scenarios for OSPF and EIGRP are configured with three selected layer 2 technologies ATM, FDDI and

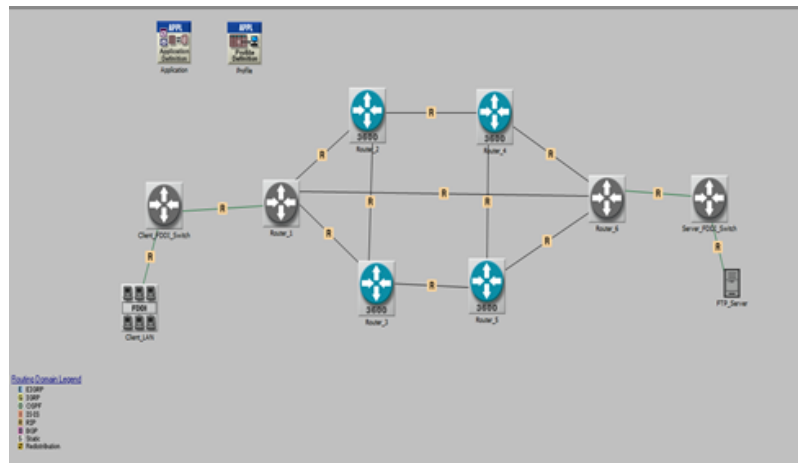

Fig. 5. Simulation RIP with FDDI

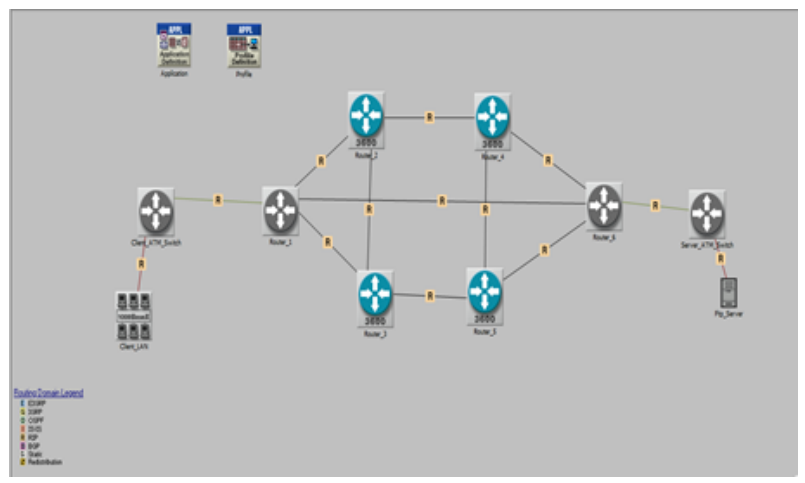

Fig. 6. Simulation RIP with ATM

Ethernet.

\section{B. Simulation Environment Parameters}

Table 2 provide a detail overview of simulation environment parameters which include protocols used, application type, Devices used, link capacity, performance measuring parameters and simulation. Except routing protocols and layer

TABLE II. SCENARIO ENVIRONMENT PARAMETERS

\begin{tabular}{|l|l|}
\hline Protocol & RIP, OSPF, EIGRP, Ethernet, FDDI, ATM \\
\hline Application & FTP (High Load) \\
\hline Switch Type & CISCO 3640 \\
\hline No of Routers & 6 \\
\hline Link Capacity & Layer 3 Links (DS1 1.54Mbps) \\
\hline & Ethernet 1000Mbps, ATM, FDDI Links \\
\hline Performance Measuring Parameters & Ethernet Delay, Routing Traffic Received/ Sent \\
\hline & Routing Traffic Received/ Sent \\
\hline & FTP Download/Upload Response Time \\
\hline & FTP Traffic Sent/Received \\
\hline & FTP Traffic Received \\
\hline Lim Time & Network Convergence Activity \\
\hline & Network Convergence Duration \\
\hline & 50 Nodes \\
\hline & 1 Hour \\
\hline
\end{tabular}


2 technologies all other simulation related parameters remains constant in all 9 constructed scenarios. The table contains constant and variable configuration parameters and performance measuring parameters.

\section{RESUlTS AND DisCUSSION}

In this chapter simulation results produced by scenarios are placed and discussed. In first part comparison between the performance of selected protocols RIP, EIGRP and OSPF are analyzed using performance measuring parameters related to routing like Routing Traffic Sent/Received, Network Convergence Activity and Duration. In second section results of FTP application services are discussed for performance analysis of the FTP server and FTP client. Performance analysis of different medium access layer technologies is performed to analyze the performance of routing protocols with conjunction of layer 2 mediums.

\section{A. Routing Protocol Performance Comparison}

In this section routing protocols RIP, EIGRP and OSPF are analyzed with three different medium access layer technologies Ethernet, ATM and FDDI. Results in this section elaborate how selected routing protocols behave with different layer 2 technologies. Selected layer 2 technologies operate entirely different in their domains as Ethernet on Medium Access layer sent packets of variable lengths and transportation of packets is performed over copper medium. ATM on same medium access layer provides fixed length data segments while FDDI provides data transportation over fiber distributed interface. Following are results of routing protocols for performance comparison used with different layer 2 technologies.

\section{B. Routing Traffic Received/Sent (Bits/Second)}

Routing traffic Received/Sent parameters set for the comparison of RIP, EIGRP, and OSPF with the layer 2 technologies to observe routing traffic generated by routing protocols.

1) Routing Traffic Sent (RIP, EIGRP, OSPF with Ethernet): Fig. 7 elaborate comparison of Routing Traffic Sent of three routing protocols with Ethernet. At start of simulation OSFP routing protocol sent bulk traffic for routing information with adjacent nodes and max traffic of OSPF at start of simulation can observed up to $5500 \mathrm{bits} / \mathrm{sec}$ while other two routing protocols EIGRP and RIP send low traffic compare to OSPF. Once network convergence performed successfully OSPF and EIGRP decreases their routing traffic while RIP constantly sent routing information. This constant routing traffic sent of RIP is because RIP periodically broadcasts its routing table information to adjacent nodes.

2) Routing Traffic Sent (RIP, EIGRP, OSPF with ATM): Fig. 8 depicts a comparison of routing protocols traffic sent with ATM transmission. It can examined that OSPF at start of simulation sent routing information with 5900 bits/Sec. EIGRP and RIP start their routing information from 3000 bits/sec and $2600 \mathrm{bits} / \mathrm{sec}$ respectively. With execution of simulation time, both OSPF and EIGRP decrease their routing information traffic while RIP constantly sent its routing information with adjacent nodes and the reason is same mentioned in previous comparison of routing information sent with Ethernet.

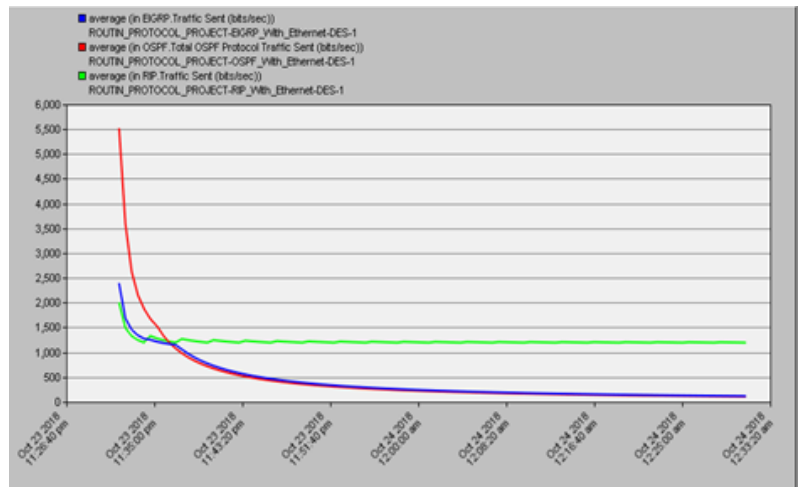

Fig. 7. Routing Traffic Sent (RIP, EIGRP, and OSPF with Ethernet)

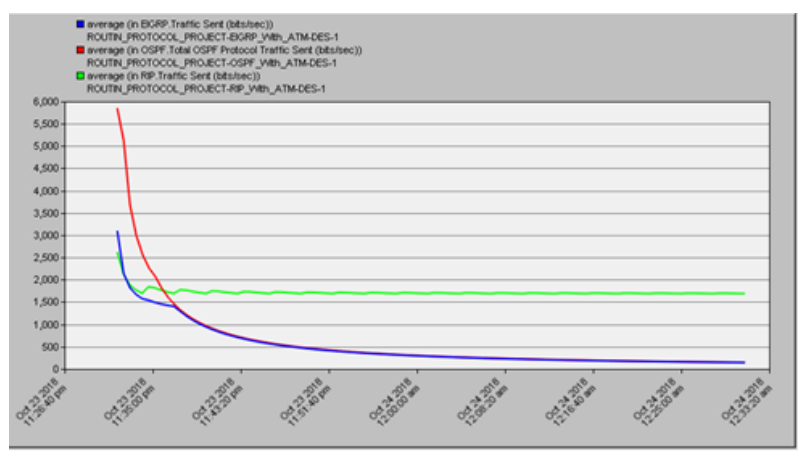

Fig. 8. Routing Traffic Sent (RIP, EIGRP and OSPF with ATM)

3) Routing Traffic Sent (RIP, EIGRP, OSPF with FDDI: Fig. 9 explained Routing Traffic Sent comparison for selected routing protocols with FDDI. At start of simulation, OSPF starts routing information traffic from $5700 \mathrm{bits} / \mathrm{sec}$ and EIGRP from 3000 bits/sec but both suddenly decrease their routing traffic once network is converged. EIGRP and OSPF sent very low routing traffic throughout simulation while RIP as oppose to EIGRP and OSPF sent routing table information to its adjacent nodes. It can observe that RIP with Ethernet and ATM sent constant routing information while with FDDI variation in routing traffic sent can be figured out that is because of highspeed links of FDDI that provide high-speed transmission.

Routing traffic received parameter set for the comparison of RIP, EIGRP, and OSPF with the layer 2 technologies that provides information about routing traffic received using

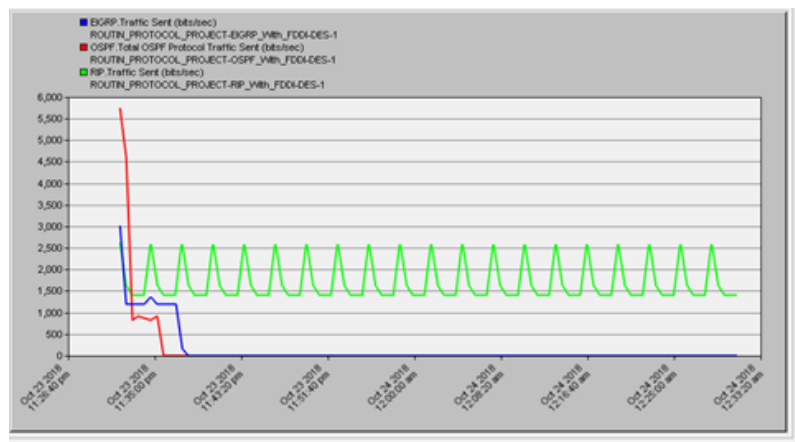

Fig. 9. Routing Traffic Sent (RIP, EIGRP, and OSPF with FDDI) 


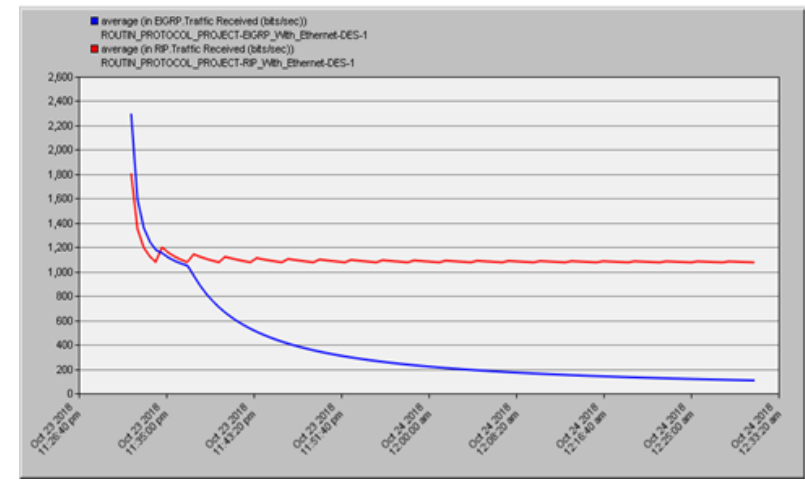

Fig. 10. Routing Traffic Received (RIP, EIGRP, OSPF with Ethernet)

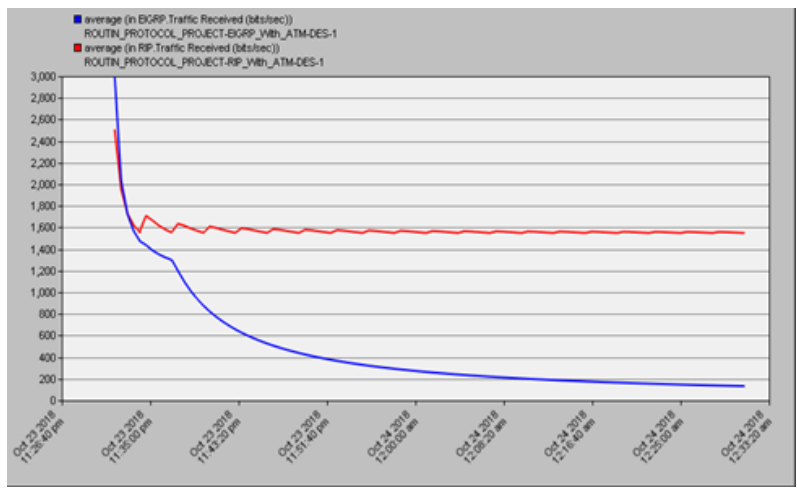

Fig. 11. Routing Traffic Received (RIP, EIGRP, OSPF with ATM)

routing protocols.

4) Routing Traffic Received (RIP, EIGRP, OSPF with Ethernet): Fig. 10 describes the result analysis of Routing Traffic Received for selected routing protocols and layers 2 technologies. Above graph depicts that at start of simulation EIGRP with Ethernet starts traffic from $2300 \mathrm{bit} / \mathrm{sec}$ and gradually with execution of simulation routing traffic decreased up to 100 $\mathrm{bit} / \mathrm{sec}$. Routing traffic of RIP with Ethernet started from 1800 $\mathrm{bit} / \mathrm{sec}$ and decreased to $1100 \mathrm{bit} / \mathrm{sec}$ throughout the simulation routing traffic received of RIP with Ethernet can be observed constant. This behavior of constant routing traffic of RIP with Ethernet is due to periodically broadcast of routing table that RIP sent to adjacent nodes.

5) Routing Traffic Received (RIP, EIGRP, OSPF with $A T M)$ : Fig. 11 describe routing traffic received of three routing protocols with ATM transmission method. In previous and following graphs it can observed that OSPF does not receive routing traffic from its adjacent nodes it is because once network with OSPF has been converged OSPF send routing information to its adjacent nodes when change in network topology occurred. In following graph EIGRP with ATM starts it routing traffic from $3000 \mathrm{bits} / \mathrm{sec}$ and progressively decrease up to $100 \mathrm{bits} / \mathrm{sec}$ while RIP with ATM starts receiving routing traffic from $2500 \mathrm{bits} / \mathrm{sec}$ and after convergence of network decreased up to $1600 \mathrm{bit} / \mathrm{sec}$ that remained constant throughout simulation time.

6) Routing Traffic Received (RIP, EIGRP, OSPF with $F D D I$ ): Above Fig. 12 elaborate routing traffic received of RIP, EIGRP, and OSPF with FDDI. In combination of EIGRP with

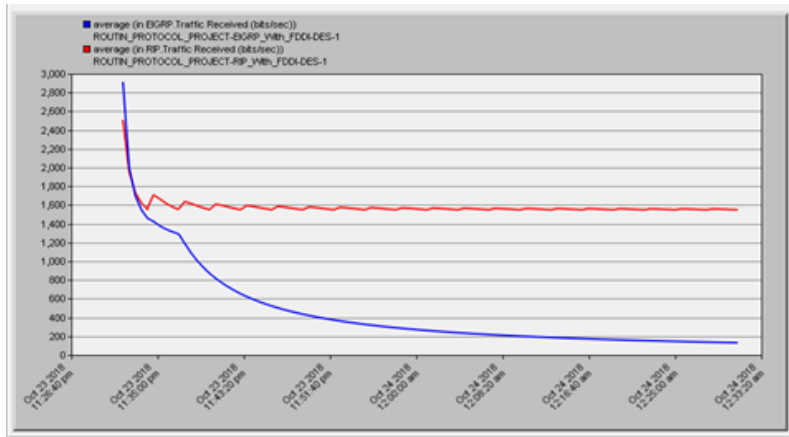

Fig. 12. Routing Traffic Received (RIP, EIGRP, OSPF with FDDI)

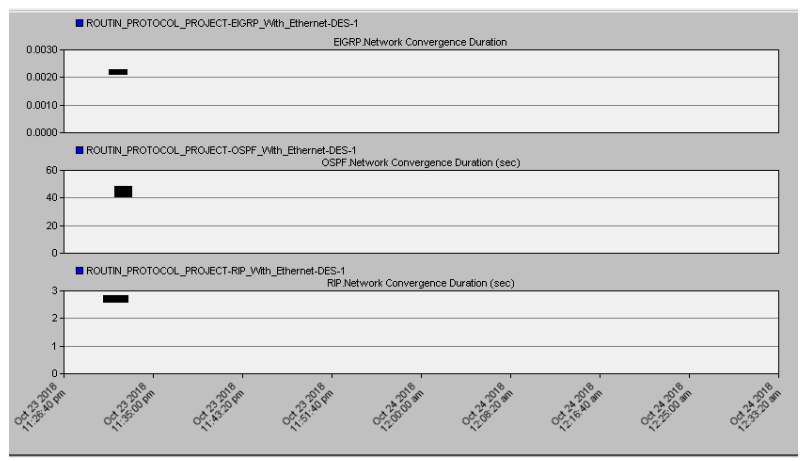

Fig. 13. Network Convergence Duration (RIP, EIGRP and OSPF with Ethernet

FDDI routing traffic received started from $2900 \mathrm{bits} / \mathrm{sec}$ and reduced up to $100 \mathrm{bits} / \mathrm{sec}$ but the combination RIP with FDDI routing traffic received graph depict starting traffic from 2500 bits/sec and remained same until the simulation termination with $1600 \mathrm{bits} / \mathrm{sec}$.

\section{Network Convergence Duration}

Network Convergence duration parameter set for the comparison of RIP, EIGRP, and OSPF with the layer 2 technologies.

1) Network Convergence Duration (RIP, EIGRP and OSPF with Ethernet): Fig. 13 illustrate comparison of Network Convergence Duration for selected routing protocols with Ethernet medium. OSPF with Ethernet take maximum time for network convergence reason can be OSPF bulk routing information that OSPF send for network convergence. Hello packets from each node configured with OSPF will send its routing table to adjacent nodes. EIGRP with Ethernet consume minimum time for network convergence and RIP with Ethernet provides better network convergence time then OSPF but not well than EIGRP.

2) Network Convergence Duration (RIP, EIGRP, and OSPF with ATM): Above Fig. 14 illustrate network convergence duration for routing protocols with ATM transmission media. Like previous result with Ethernet same in this scenario OSPF with ATM consumed maximum time for network convergence that is $55 \mathrm{sec}$, RIP provides $10 \mathrm{sec}$ for network convergence and EIGRP with ATM showed best performance and provides least network convergence time. 


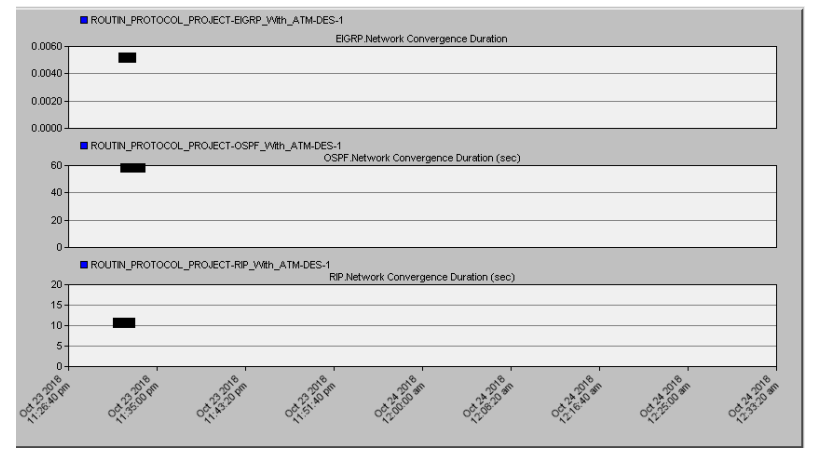

Fig. 14. Network Convergence Duration (RIP, EIGRP, and OSPF with ATM

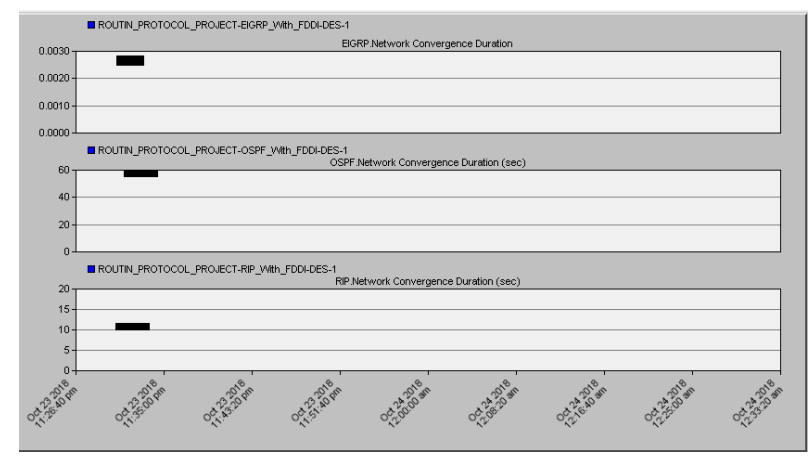

Fig. 15. Network Convergence Duration (RIP, EIGRP, and OSPF with FDDI

3) Network Convergence Duration (RIP, EIGRP, and OSPF with FDDI): Fig. 15 provide comparison of routing protocols for network convergence duration, OSPF with FDDI behaves same as it behaves with Ethernet and ATM and provide higher network convergence time. RIP with FDDI also provide higher network convergence time than EIGRP and it is due to its periodically updates mechanism. EIGRP is protocol which provide least network convergence time with all three layers 2 transmission technologies.

4) Network Convergence Duration (Ethernet, ATM and FDDI with RIP): Above Fig. 16 elaborate network convergence time RIP protocol with three different layers 2 mediums. For network convergence RIP with FDDI consume higher network convergence time that is $10.5 \mathrm{sec}$ and RIP with ATM provide lesser network convergence time than RIP with FDDI. Above Figure clarified that RIP with Ethernet provides

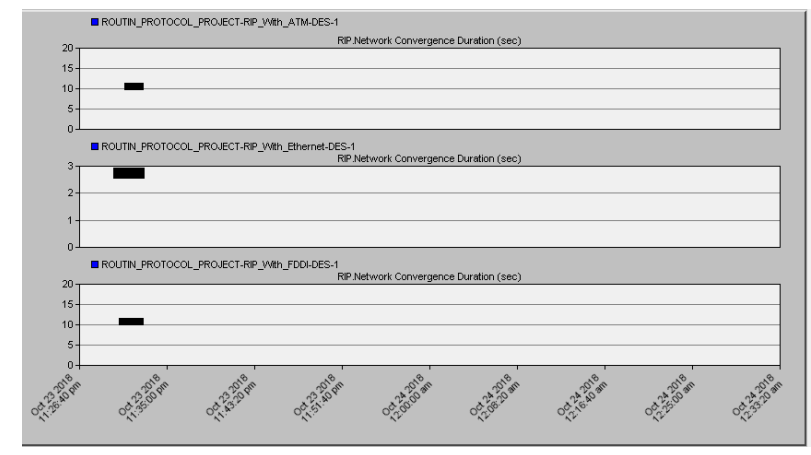

Fig. 16. Ethernet, ATM and FDDI with RIP

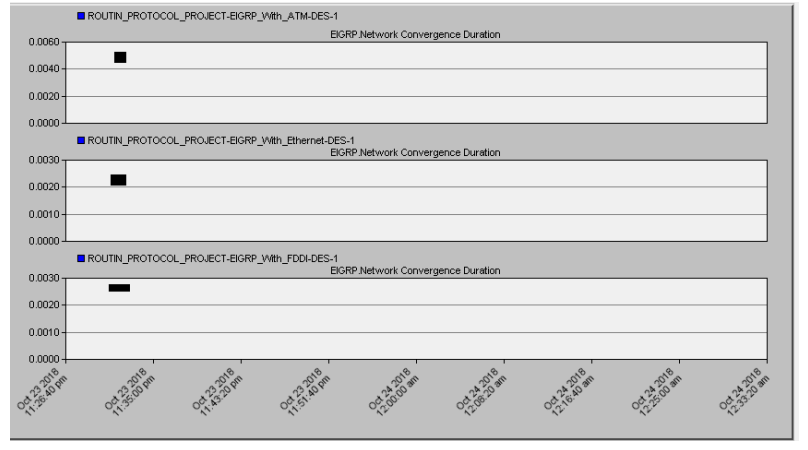

Fig. 17. Ethernet, ATM and FDDI with EIGRP

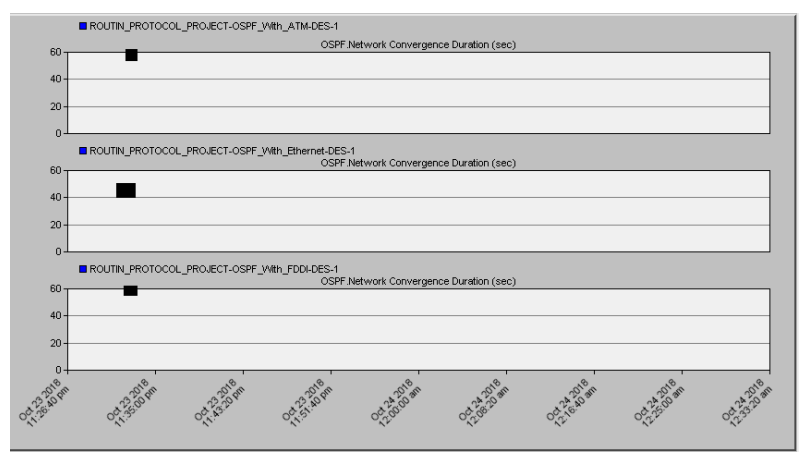

Fig. 18. Ethernet, ATM and FDDI with OSPF

least network convergence time that is $2.8 \mathrm{sec}$. This graph elaborate that RIP when configure with Ethernet it provide lowest network convergence time than ATM and FDDI.

5) Network Convergence Duration (Ethernet, ATM and FDDI with EIGRP): In Fig. 17 Network convergence duration of EIGRP with three layers 2 transmission mediums are described in above graph. EIGRP with Ethernet provides lowest network convergence time while EIGRP with ATM and FDDI provides higher network convergence time than Ethernet. This shows that when EIGRP configured with Ethernet, ATM and FDDI mediums it behaves well with Ethernet with respect to network convergence time.

6) Network Convergence Duration (Ethernet, ATM and FDDI with OSPF): Fig. 18 compares network convergence duration for OSPF with ATM, Ethernet and FDDI transport medium. OSPF with ATM and FDDI provides same network convergence duration that is $57 \mathrm{sec}$ while OSPF with Ethernet provides lowest network convergence time that is $45 \mathrm{sec}$. This graph result depicts that OSPF has good collaboration with Ethernet layer 2 medium when network convergence duration is being analyzed.

\section{FTP Client Download/Upload Response Time}

FTP client download/upload response time parameters set for the comparison of RIP, EIGRP, and OSPF with the layer 2 technologies. Different combinations are given below.

1) FTP Client Download Response Time (RIP, EIGRP, and OSPF with Ethernet): Above mentioned Fig. 19 elaborate comparison of FTP Client download response time of three routing protocols with Ethernet. At start of simulation OSFP 


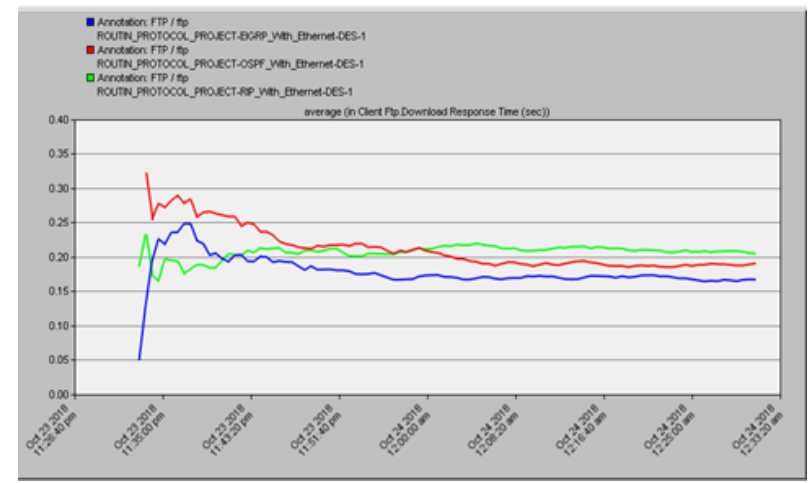

Fig. 19. FTP Client Download Response Time (RIP, EIGRP and OSPF with Ethernet)

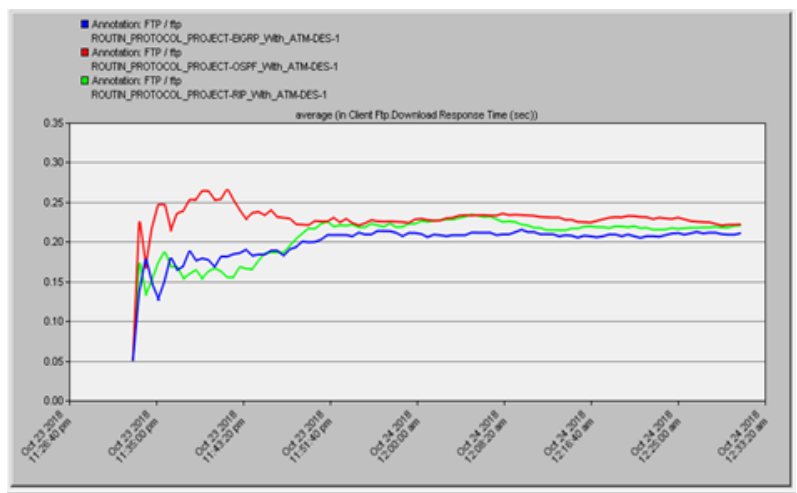

Fig. 20. FTP Client Download Response Time (RIP, EIGRP and OSPF with ATM)

download response time shows higher than others protocols $0.35 \mathrm{bits} / \mathrm{sec}$ but with simulation execution it is observed up to 0.17 bits/sec. EIGRP and RIP start with low download response time compare to OSPF. But Ethernet with RIP shows better performance than other 2 protocols. OSPF and EIGRP start download response time $0.18 \mathrm{bits} / \mathrm{sec}$ and remain comparatively same end up with $0.21 \mathrm{bits} / \mathrm{sec}$. Performance of RIP with Ethernet is better than others.

2) FTP Client Download Response Time (RIP, EIGRP, and OSPF with ATM): Fig. 20 elaborate comparison of FTP Client download response time of routing protocols with ATM. At start of simulation OSFP and EIGRP download response time shows lesser and equal than other protocol $0.5 \mathrm{bits} / \mathrm{sec}$ but with simulation time witnessed download response time of OSPF, EIGRP and RIP up to $0.21 \mathrm{bits} / \mathrm{sec}$ while RIP start with download response time high compare to others. ATM with RIP show better performance than other protocols with starting download response time $0.16 \mathrm{bits} / \mathrm{sec}$ and remain comparatively same end up with $0.21 \mathrm{bits} / \mathrm{sec}$. You can say that performance of RIP with ATM is better than others.

3) FTP Client Download Response Time (RIP, EIGRP and OSPF with FDDI): Fig. 21 illustrate comparison of FTP Client download response time of three routing protocols with FDDI network. All routing protocols behave same throughout simulation time.

4) FTP Client Upload Response Time (RIP, EIGRP and OSPF with Ethernet): In Fig. 22 comparison of FTP Client

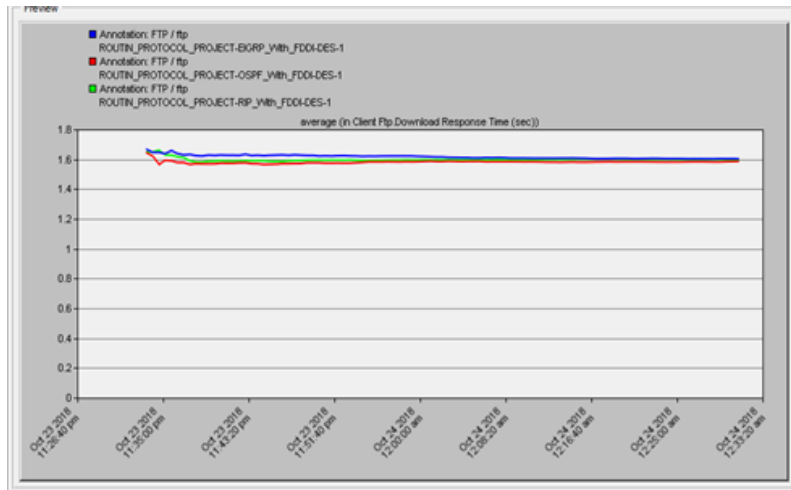

Fig. 21. FTP Client Download Response Time (RIP, EIGRP and OSPF with FDDI)

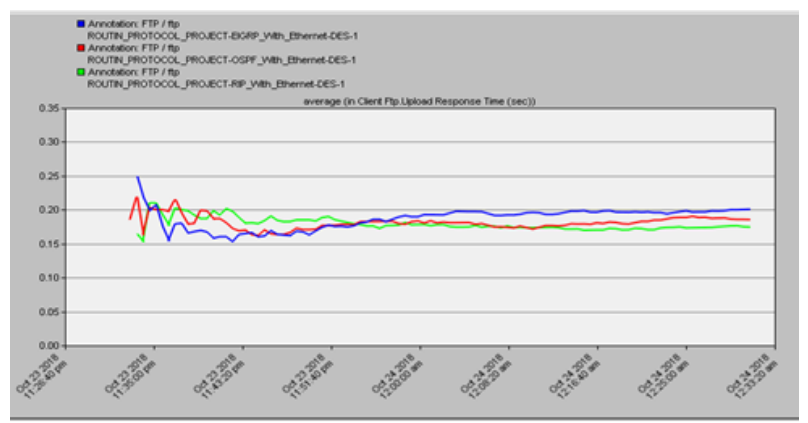

Fig. 22. FTP Client Upload Response Time (RIP, EIGRP and OSPF with Ethernet)

Upload response time with three routing protocols when configured with Ethernet. Graphs conclude that FTP client upload response time for EIGRP with Ethernet was low at start of simulation while with execution of simulation it goes higher. At start it was 0.25 when the network was being converged after convergence this FTP client upload response time decrease up to $0.15 \mathrm{sec}$ and at mid of simulation time it again started to increase and on 0,20 sec remained constant throughout the simulation. FTP client upload response time for OSPF and RIP with Ethernet behave almost identical throughout the simulation and depict upload response time from $0.17 \mathrm{sec}$ to $0.20 \mathrm{sec}$.

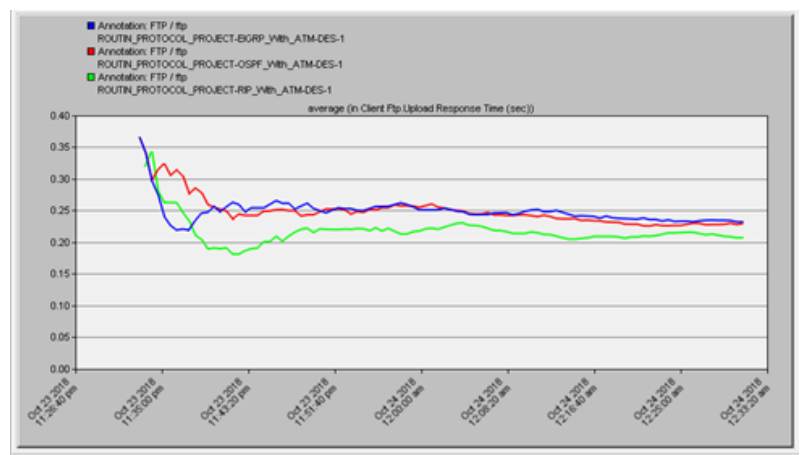

Fig. 23. FTP Client Upload Response Time (RIP, EIGRP and OSPF with ATM) 


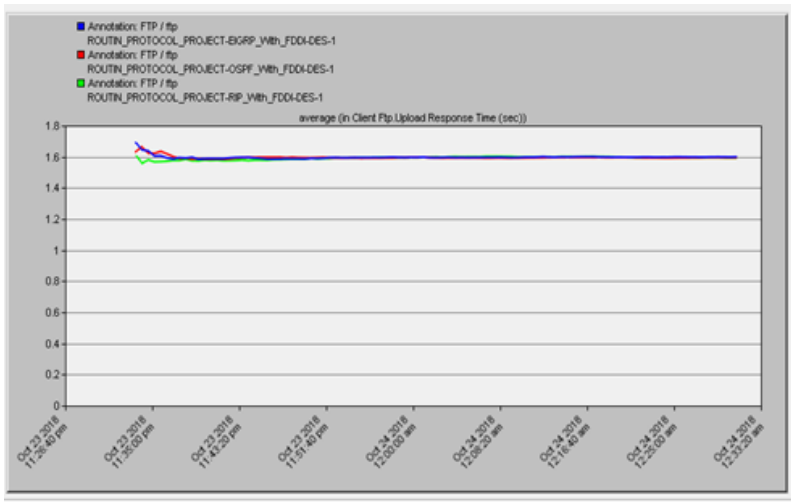

Fig. 24. FTP Client Upload Response Time (RIP, EIGRP and OSPF with FDDI)

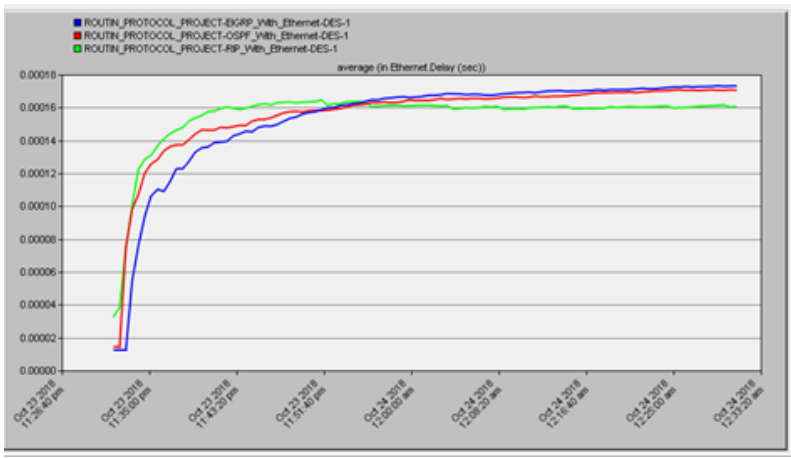

Fig. 25. ETHERNET DELAY (RIP, EIGRP and OSPF with Ethernet)

5) FTP Client Upload Response Time (RIP, EIGRP and $O S P F$ with ATM): Above result of Fig. 23 describe comparison of routing protocols with ATM. This graph described that at start of the simulation when all three routing protocols were sharing their routing information FTP client upload response time was higher but with simulation execution RIP with ATM provide lowest FTP client upload response time but OSPF and EIGRP with ATM provide comparatively higher FTP client upload response time than RIP with ATM. RIP with ATM work well with reference to FTP client upload response time.

6) FTP Client Upload Response Time (RIP, EIGRP and OSPF with FDDI): FTP client upload time comparison for three routing protocols with FDDI medium is illustrated above. Above graph in Fig. 24 shows with FDDI all routing protocols behave same with reference to FTP client upload response time. RIP, OSPF and EIGRP with FDDI provide same ftp upload response time that is $1.8 \mathrm{sec}$.

\section{ETHERnet Delay (SECONDS)}

Ethernet Delay performance measuring parameters is observed for monitoring of routing protocol performance with layer 2 mediums and packet delay at layer 2 medium.

1) Ethernet Delay (RIP, EIGRP and OSPF with Ethernet): Fig. 25 illustrates comparison of routing protocols RIP, OSPF and EIGRP with Ethernet for calculation of Ethernet delay. The graph represents that with start of simulation EIGRP provide low Ethernet delay than OSPF and RIP. At the start of the simulation OSPF and RIP Ethernet delay were higher but at the

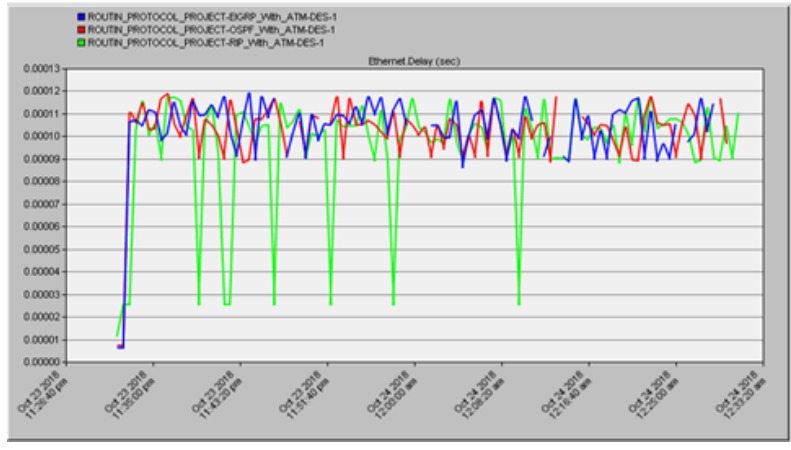

Fig. 26. Ethernet Delay (RIP, EIGRP and OSPF with ATM)

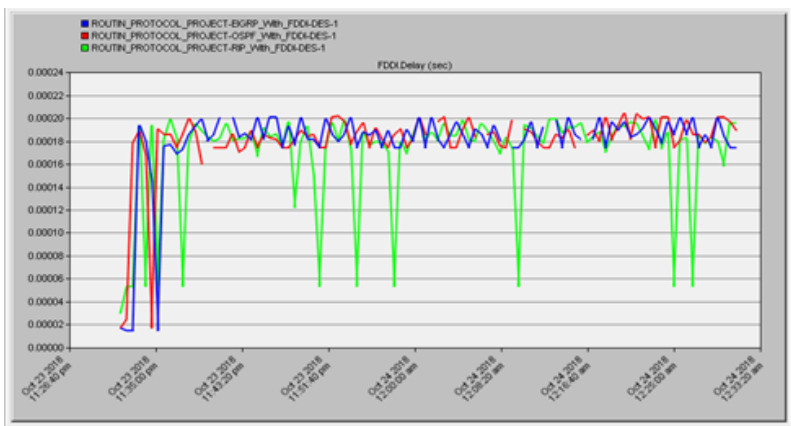

Fig. 27. FDDI DELAY (RIP, EIGRP and OSPF with FDDI)

mid of simulation RIP with Ethernet medium decreased and throughout simulation remain constant. After half execution of simulation time OSPF and RIP with Ethernet increases their Ethernet delay and this is because OSPF especially sends higher routing traffic.

2) Ethernet Delay (RIP, EIGRP and OSPF with ATM): Fig. 26 illustrate relationship between routing protocols and ATM transmission medium for Ethernet delay. Graph elaborate that RIP with ATM provide low Ethernet delay that is recorded $0.00025 \mathrm{sec}$. OSPF and EIGRP with ATM provide higher Ethernet delay than RIP with ATM. This concludes that when RIP is configured with ATM as layer 2 medium it provides lowest Ethernet delay and perform best with respect to latency time.

3) FDDI Delay (RIP, EIGRP and OSPF with FDDI): Fig. 27 represents a comparison of routing protocol RIP, EIGRP and OSPF with FDDI medium. At the start of simulation three protocols provide same Ethernet delay with FDDI transport medium. After the execution of the simulation, it can be observed that RIP with FDDI provide low Ethernet delay while other two routing protocols EIGRP and OSPF behaves same for FDDI medium and acts more delay prone then RIP when configured with FDDI.

4) Ethernet Delay (Ethernet, ATM and FDDI with RIP): Comparison of the RIP routing protocol with three layers 2 mediums for analyzing the Ethernet delay is represented in the above-mentioned graph of Fig. 28. Graph elaborate that RIP with ATM medium provide the least delay that is recorded $0.00008 \mathrm{sec}$ to $0.0009 \mathrm{sec}$ and it was very least at the starting of the simulation that is $0.00002 \mathrm{sec}$. RIP with other two layers 2 mediums provides higher Ethernet delay. RIP with 


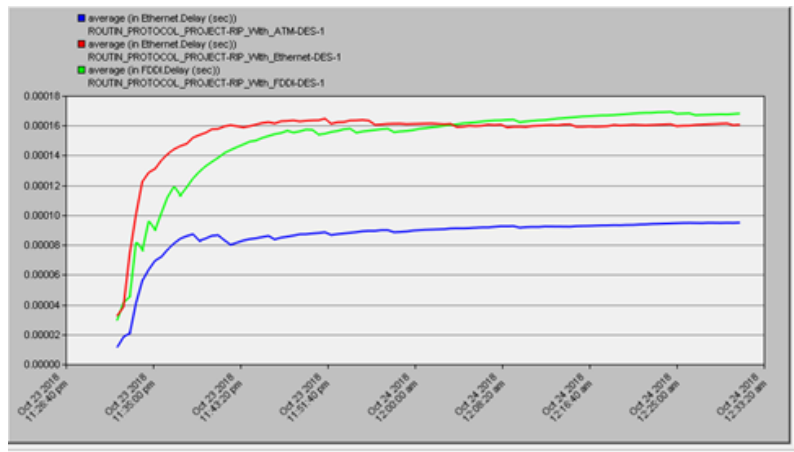

Fig. 28. Ethernet Delay (Ethernet, ATM and FDDI with RIP)

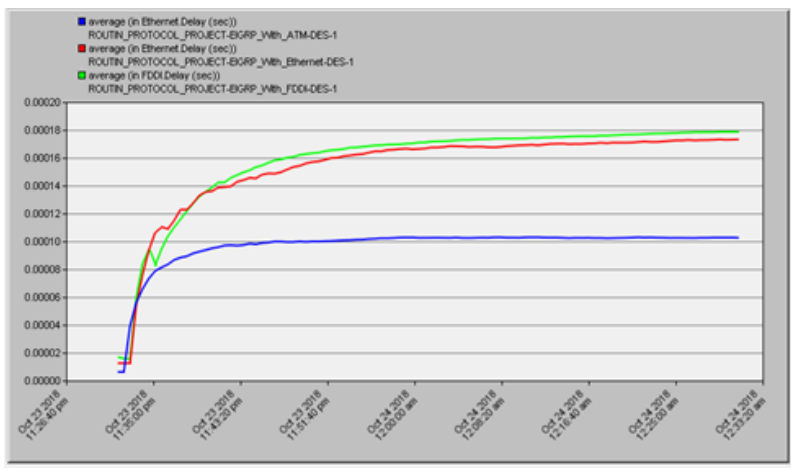

Fig. 29. Ethernet Delay (Ethernet, ATM and FDDI with EIGRP)

ATM provide $0.00016 \mathrm{sec}$ and this delay was at the starting of the simulation was $0.00004 \mathrm{sec}$ that remained constant at the level of $0.00016 \mathrm{sec}$ throughout the simulation. RIP with FDDI start with Ethernet delay $0.00004 \mathrm{sec}$ and at the mid of the simulation this Ethernet delay increased up to $0.00017 \mathrm{sec}$. This graph result describes that RIP with ATM performs best.

5) Ethernet Delay (Ethernet, ATM and FDDI with EIGRP): Above mentioned result graph elaborate that EIGRP how to behave with ATM, Ethernet and FDDI layer 2 mediums. This Fig. 29 described that EIGRP with ATM layer 2 technology provide lowest Ethernet delay that is observed at the starting of the simulation $0.00005 \mathrm{sec}$ and remain constant at 0.00010 sec throughout the simulation. EIGRP with ATM and Ethernet provide higher Ethernet delay that is observed up to 0.00014 $\mathrm{sec}$ to $0.00014 \mathrm{sec}$ for EIGRP with ATM and FDDI. EIGRP, when configured with ATM, provide lowest packet delay than other two layers 2 transmission mediums.

6) Ethernet Delay (Ethernet, ATM and FDDI with OPSF): Fig. 30 illustrates comparison of OSPF routing protocol with three layer 2 transmission mediums. Above graph elaborate that OSPF with ATM provides low Ethernet delay and OSPF with ATM and Ethernet provides the higher delay. Though it is observed in previous results that OSPF is more routing traffic generated protocols and has higher routing overhead over bandwidth links when OSPF configured with ATM it provides lowest Ethernet delay. OSPF with Ethernet and ATM provide Ethernet delay from $0.00016 \mathrm{sec}$ to $0.00018 \mathrm{sec}$ but OSPF with ATM provide $0.00002 \mathrm{sec}$ and at $0.00010 \mathrm{sec}$ this Ethernet delay became constant for throughout the simulation.

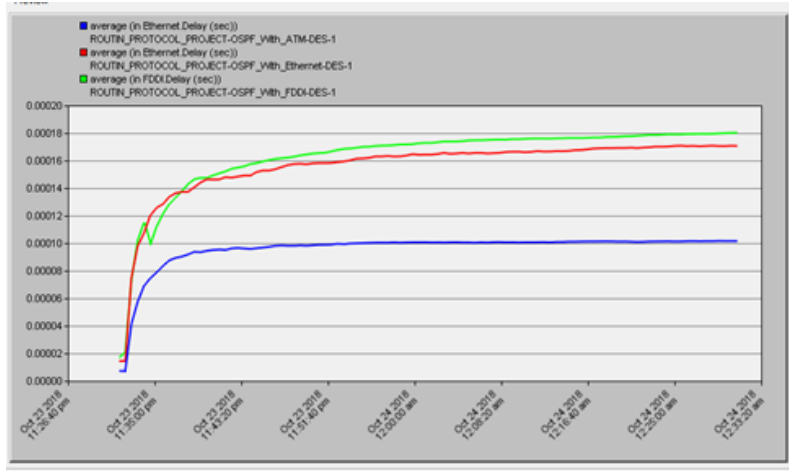

Fig. 30. Ethernet Delay (Ethernet, ATM and FDDI with OPSF)

\section{SUMMARY}

On the basis of simulation results obtained conclusion for simulation scenarios is represented in this summary. Routing traffic sent is compared for three different layers 2 transmission mediums. Routing Traffic sent for Ethernet using RIP, OSPF and EIGRP are analyzed and results described that RIP with Ethernet layer 2 medium EIGRP with Ethernet starts low routing traffic at the start of the simulation while OSPF with Ethernet at the start of the simulation started with a high routing traffic. With the execution of simulation time, OSPF with Ethernet decreased its routing traffic while RIP with Ethernet constantly sends same routing traffic throughout the simulation. EIGRP and OSPF act well and reduce their routing traffic. Constant routing traffic of RIP is due to its periodically broadcasting of routing table information. So it can conclude that RIP due to its periodically broadcasting consumes higher bandwidth utilization and an extra overhead in bandwidth consumption is observed. OSPF and EIGRP are well efficient in bandwidth consumption and only send routing traffic after network convergence when any topology change occurred. Same behavior of RIP is observed for ATM and FDDI medium. OSPF and EIGRP in start sent high routing traffic but after network convergence reduces their routing traffic that is the edge of these protocols with consideration of routing traffic sent that utilize extra bandwidth. Routing traffic sent comparison of each protocol with layer 2 medium to conclude that RIP when configured with Ethernet its sent low routing traffic then RIP with ATM and FDDI so RIP performs better with Ethernet with respect to routing traffic sent.

For routing, traffic received performance measuring parameters three routing protocols are examined with layer 2 mediums one by one and concluded that when routing protocols are configured with Ethernet layer 2 medium RIP with Ethernet transmit higher routing traffic than other routing protocols and this is also due to RIP broadcast its routing tables periodically. With ATM layer 2 medium routing protocols, EIGRP again behaves better than RIP and the reason is the same RIP periodically updates. Utilization of routing protocols with FDDI layer 2 medium illustrate that RIP again behaves worse. So with routing traffic sent and received parameters describe that RIP due to its periodical updates consumes higher bandwidth utilization then OSPF and EIGRP. When RIP is configured with three layers 2 mediums and analyzed its performance with these medium its performance with Ethernet found better than ATM and FDDI. 
For network convergence duration performance measuring parameter, first routing protocols are examined with layer 2 mediums one by one and concluded that with Ethernet OSPF consumes higher network convergence duration then EIGRP and RIP so OSPF is worse in network convergence with Ethernet. OSPF with ATM and FDDI again consume higher convergence time then EIGRP and RIP. EIGRP is routing protocols which consume minimum network convergence duration with all three layers 2 mediums so it is concluded that EIGRP takes less network convergence time without limitation of layer 2 medium. Analysis of routing protocols for network convergence duration individually with Ethernet, ATM, and FDDI show that with Ethernet RIP consume lowest network convergence duration and OSPF and EIGRP with Ethernet also consume low network convergence duration. These analyses conclude that Ethernet is best to layer 2 medium when network convergence duration is considered.

FTP client upload response time performance measuring parameter describes which routing protocol and layer 2 medium is the best fit. For this performance measuring parameter first three routing protocols are analyzed with layer 2 medium individually. Routing protocols EGIRP, RIP and OSPF when test with Ethernet it showed that EIGRP with Ethernet provides lowest FTP client upload response time then OSPF and RIP. For ATM layer 2 network EIGRP again behaves well and provide lowest ftp upload response time but for FDDI network ftp upload response time for all three routing protocols is observed the same. This depicts that FDDI network do not affect the performance of routing protocols but on Ethernet and ATM layer 2 Mediums EIGRP perform well. When routing protocols are observed for FTP client upload response time with different layer 2 mediums it showed that RIP with Ethernet and ATM provides lowest ftp upload response time but FDDI medium is not good for upload response time. EIGRP again behave worse with FDDI and provide lowest ftp upload response time with ATM and Ethernet. OSPF with ATM and Ethernet again behaves best with consideration of FTP upload response time. Analysis of this performance measuring parameters concludes that FDDI medium is more delay prone with reference to ftp upload response time and ATM and Ethernet layer 2 mediums are best for routing protocols. When routing protocols are analyzed with layer 2 mediums for FTP upload response time it concluded that RIP with Ethernet and ATM provide lowest ftp upload response time but with FDDI all routing protocols behave same. Analysis for FTP download response time are also performed and found the same behavior of routing protocols as with FTP upload response time.

For Ethernet delay to monitor the effect of routing protocols and layer 2 medium over latency is performed. First selected routing protocols are examined with Ethernet medium and found that RIP with Ethernet at the starting of the simulation provides higher Ethernet delay but with simulation execution RIP reduced its Ethernet delay then OSPF and EIGRP. Over ATM layer 2 medium RIP provide lowest Ethernet delay and OSPF and EIGRP behave identically. With FDDI layer 2 medium again RIP show low delay prone routing protocol then OSPF and EIGRP. Analysis of routing protocols for packet latency with the conjunction of different layer 2 mediums concluded that when the RIP is configured with ATM layer 2 medium it provides lowest latency rate and EIGRP with ATM also provides lowest Ethernet delay and same for OSPF that provide low Ethernet delay with ATM then Ethernet and FDDI. Routing protocols configured with layer 2 medium for packet latency and Ethernet delay concluded that ATM layer 2 medium and RIP routing protocol is the best fit when used to analyze latency rate so it can be concluded that when network with low Ethernet delay and latency rate are required RIP from routing protocols and ATM from layer 2 mediums are best choice.

\section{REFERENCES}

[1] Soorki, M. N., and Rostami, H. (2014). Label switched protocol routing with guaranteed bandwidth and end to end path delay in MPLS networks. Journal of Network and Computer Applications, 42, 21-38.

[2] Wijekoon, J., Harahap, E., \& Nishi, H. (2013). Service-oriented router simulation module implementation in ns 2 simulator. Procedia Computer Science, 19, 478-485.

[3] Nixon, S., \& Dana, D. (2017). Modeling Network Optimization by Optimize the Current Network by physical and logical architectures to improve the Quality of Services (QoS). International Journal of Engineering Science, 14715.

[4] Wolf, T., and Turner, J. S. (2001). Design issues for high-performance active routers. IEEE Journal on Selected Areas in Communications, 19(3), 404-409.

[5] Ravi, G., and Kashwan, K. R. (2015). A new routing protocol for energy efficient mobile applications for ad hoc networks. Computers and Electrical Engineering, 48, 77-85.

[6] Omitola, O. O. (2014). Evaluation and Investigation of Throughput and Delay on Ethernet and FDDI Technologies using OPNET. Pacific Journal of Science and Technology, 15(1), 125-129.

[7] Filipiak, J., and Chemouil, P. (1991, December). Routing and bandwidth management options in high speed integrated services networks. In Global Telecommunications Conference, 1991. GLOBECOM'91.'Countdown to the New Millennium. Featuring a Mini-Theme on: Personal Communications Services (pp. 1685-1689). IEEE.

[8] Fiţigău, I., and Toderean, G. (2013, June). Network performance evaluation for RIP, OSPF and EIGRP routing protocols. In Electronics, Computers and Artificial Intelligence (ECAI), 2013 International Conference on (pp. 1-4). IEEE.

[9] https://www.imedita.com/blog/distance-vector-routing-protocols/

[10] (http://www.cisco.com/en/US/docs/internetworking/)

[11] https://slideplayer.com/slide/8946913/

[12] Pamies-Juarez, L., Datta, A., and Oggier, F. (2013). In-network redundancy generation for opportunistic speedup of data backup. Future Generation Computer Systems, 29(6), 1353-1362.

[13] Vetriselvan, V., Patil, P. R., and Mahendran, M. (2014). Survey on the RIP, OSPF, EIGRP routing protocols. IJCSIT) international journal of computer science and information technologies, 5(2), 1058-1065.

[14] Abdulkadhim, M. (2015). Routing Protocols Convergence Activity and Protocols Related Traffic Simulation With It's Impact on the Network. International Journal of Science, Engineering and Computer Technology, 5(3), 40.

[15] Filipiak, J., and Chemouil, P. (1991, December). Routing and bandwidth management options in high speed integrated services networks. In Global Telecommunications Conference, 1991. GLOBECOM'91.'Countdown to the New Millennium. Featuring a Mini-Theme on: Personal Communications Services (pp. 1685-1689). IEEE.

[16] Fiţigău, I., and Toderean, G. (2013, June). Network performance evaluation for RIP, OSPF and EIGRP routing protocols. In Electronics, Computers and Artificial Intelligence (ECAI), 2013 International Conference on (pp. 1-4). IEEE.

[17] Thorenoor, S. G. (2010, April). Dynamic routing protocol implementation decision between EIGRP, OSPF and RIP based on technical background using OPNET modeler. In Computer and Network Technology (ICCNT), 2010 Second International Conference on (pp. 191-195). IEEE. 
[18] Handley, M., Raiciu, C., Agache, A., Voinescu, A., Moore, A. W., Antichi, G., and Wójcik, M. (2017, August). Re-architecting datacenter networks and stacks for low latency and high performance. In Proceedings of the Conference of the ACM Special Interest Group on Data Communication (pp. 29-42). ACM.

[19] Shah, A., and Rana, W. J. (2013). Performance Analysis of RIP and
OSPF in Network using Opnet. International Journal of Computer Science Issues (IJCSI), 10(6), 256.

[20] Perez, G. E., and Kostanic, I. (2014). Comparing a Real-Life WSN Platform Small Network and its OPNET Modeler model using Hypothesis Testing. Journal on Systemics, Cybernetics and Informatics: JSCI, 12(7), 66-73. 